\title{
Two New Eisenia Species from South Korea Similar to E. koreana and Comparable to Eisenoides from USA (Oligochaeta: Lumbricidae)
}

\author{
Robert J. Blakemore*, Tae Seo Park \\ National Institute of Biological Resources (NIBR), Incheon 404-708, Korea
}

\begin{abstract}
New Korean lumbricids are described: Eisenia gaga Blakemore sp. nov. from remote Gageodo Island and E. sindo Blakemore, sp. nov. from an island at Incheon. Both are comparable to Eisenia koreana (Zicsi, 1972) from near Pyongyang, North Korea. A remarkable yet previously unrecognized similarity to American earthworm Eisenoides carolinensis (Michaelsen, 1910) is discussed, but synonymy is rejected on tenuous morphological grounds (form of nephridial vesicle bladders) with taxonomy supported by objective molecular data (mtDNA cytochrome c oxidase subunit 1 barcodes).
\end{abstract}

Keywords: Asian biodiversity, soil fauna, endemic invertebrates vs. invasive species

\section{INTRODUCTION}

Lumbricidae is a relatively large holarctic family of mostly terrestrial earthworms comprising approximately 670 valid taxa from a total of 1,130 names in 63 genera (Blakemore, 2008a). Natural distribution is from North America (e.g., Bimastos lawrenceae Fender, 1994 on Vancouver Island, Canada) throughout boreal Europe and the Middle East to far-eastern Asia (e.g., Eisenia anzac Blakemore, 2011 from Japan). Commonly transported lumbricids comprise just 33 species from a total of about 150 other cosmopolitan earthworms as detailed in Blakemore (2010). Of approximately 100 earthworm species currently known from Korea (Blakemore, 2008b), a minority dozen are Lumbricidae, most introduced exotics, some shared by neighbouring countries. Only Eisenia koreana (Zicsi, 1972) is thought wholly endemic, albeit unrecorded for the last 40 years. Here two new Korean lumbricids are described that are comparable but separable genetically, leaving E. koreana as yet unconfirmed.

Specimens, lodged in National Institute of Biological Resources (NIBR), are described in the author's usual style (e.g. Blakemore, 2010). Cytochrome c oxidase subunit 1 (COI barcode) sequences are appended with analyses via megaBLAST (www.blast.ncbi.nlm.nih.gov/BLAST.cgi). Abbreviations are rhs, right hand side; lhs, left hand side; TP, tuberculae pubertates.

\section{SYSTEMATIC ACCOUNTS}

Order Megadrilacea Benham, 1890

Family Lumbricidae Rafinesque-Schmaltz, 1815

Genus Eisenia Malm, 1877 (type-species: Enterion fetidum

Savigny, 1826)

\section{Eisenia gaga sp. nov. (Figs. 1, 2)}

Material examined. Holotype, NIBR IV IV0000245509 (dissected and figured, providing DNA-WM1) (Fig. 1); eight paratypes, NIBR IV0000245510-IV0000245517 two dissected (specimen \#2 WM2) and one photographed (\#5 WM3) (Fig. 2), all fixed in 75\% EtOH. Collected 26 Jan 2012 by Park TS, Seo HY from damp leaf litter on slopes of Mt. Doksil, $34^{\circ} 4^{\prime} 32.73 \mathrm{~N}, 125^{\circ} 6^{\prime} 31.88 \mathrm{E}$; summit $639 \mathrm{~m}$, on Gageodo Island in Yellow Sea of South Korea. Found with a damaged Amynthas gageodo Blakemore, 2012 (NIBR IV0000245518) - see Blakemore et al. (2012).

Etymology. After historical name for Gageodo Island, "Gaga" meaning "beautiful."

Description. Body square in section after segment 12 with setae at each rounded corner; colour bleached in alcohol. Lengths 80-105 mm (holotype H 100, paratype P1 80, P2 100, P3 105). Segments 124-162 (H 162, P1 124, P2 145, P3 120). Prostomium epilobic, narrow. Setae closely paired. Tu- (c) This is an Open Access article distributed under the terms of the Creative Commons Attribution Non-Commercial License (http://creativecommons.org/ licenses/by-nc/3.0/) which permits unrestricted non-commercial use, distribution, and reproduction in any medium, provided the original work is properly cited.

pISSN 2234-6953 eISSN 2234-8190
*To whom correspondence should be addressed

Tel: 82-32-590-7100, Fax: 82-32-590-7250

E-mail: rob.blakemore@gmail.com 


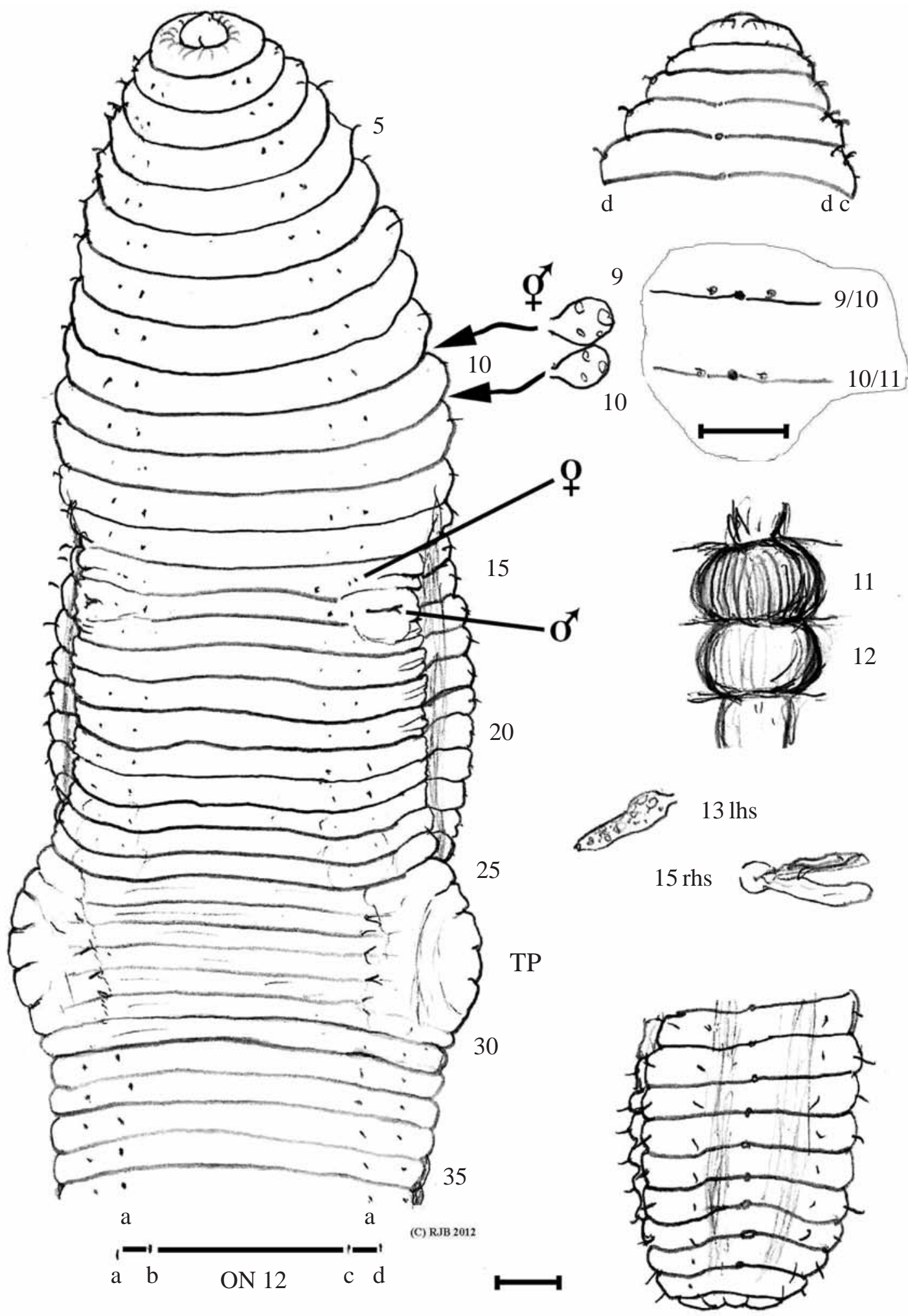

Fig. 1. Eisenia gaga: holotype (contracted specimen \#1 IV000245509) ventral view of anterior, prostomium and dorsal pores, enlargement of spermathecal and dorsal pores; spermathecae in situ, sketch of calciferous glands in 11 and 12 ovaries in 13 Ihs, nephridium in 15 rhs; posterior and pygidium, and actual setal ratios on segment 12 . rhs, right hand side; Ihs, left hand side. Scale bars $=1 \mathrm{~mm}$. 


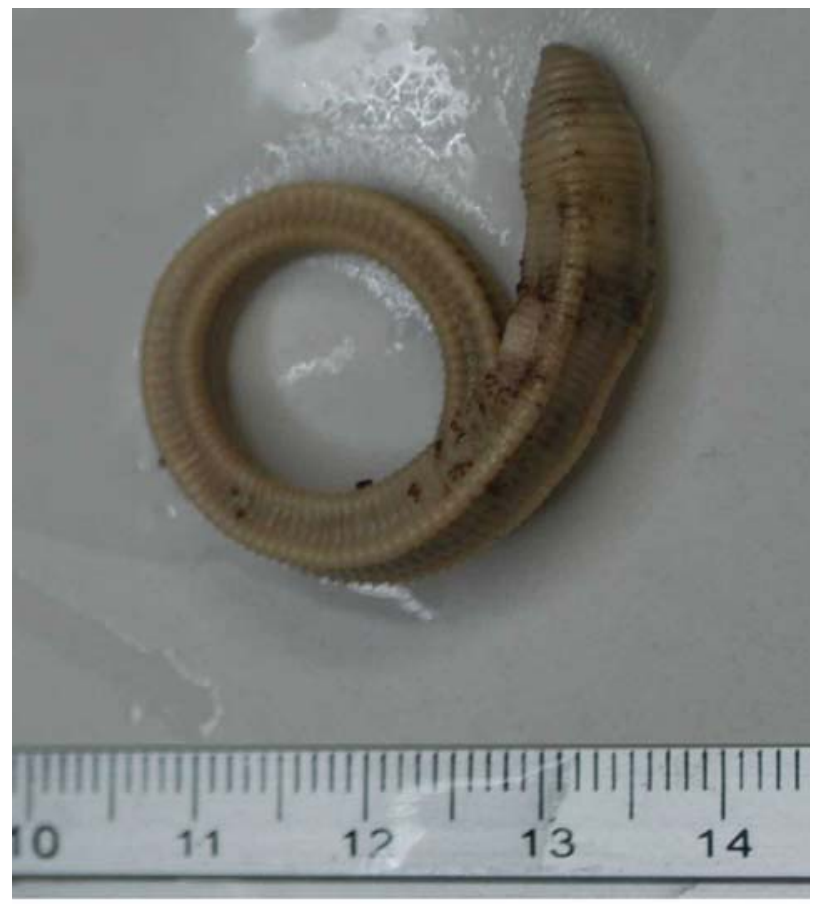

Fig. 2. Photo of dorsum of specimen \#5 (IV000245513) (by Blakemore RJ on Hanna Bae's camera).

midity obscures ventral setae in some of $11,13,14,16,17$ and 22-31 and on $10 \& 11$ laterally; ab on $14 \& 15$ are slightly displaced. Clitellum pale 24,25-30,31, typically 25-31. Tubercula pubertatis on ca. 27-29 wide of setal b line on each side. Dorsal pores from 3/4 (minute), open from 4/5. Nephropores sporadically visible above $d$ setae. Spermathecal pores in 9/10/11 close to mid-dorsal line. Female pores 14; male pores 15 with tumid mounds impinging slightly into adjacent segments.

Internally, septa 5/6-14/15 slightly thickened. Spermathecae round in 9 and 10. Testis and funnels free, iridescent in 10 and 11. Seminal vesicles in 9-12 those in (9 and) 10 smaller and filled with brown bodies. Ovaries small, flattened and tapering (like a folded hand) in 13. Ovisacs small anteriorly in 14 on 13/14. Hearts in 7-11. Nephridial bladders simple, sausage-shaped (in all segments inspected). Calciferous glands large and moniliform in 11 (vascularized) and in 12. Crop in 15 and, less dilated, in 16; muscular gizzard in 1718. Low but broad intestinal typhlosole develops from around 20-22. Gut contents, fine silty soil and organic matter and gizzard had a few largish 'crop stone' grits. Apart from 'brown bodies', no evidence of parasites was observed in the coelom, blood vessels or other organs.

Remarks. Eisenia gaga differs slightly from Zicsi's account (1972) of E. koreana where he gives shorter biometry and pro-epilobous prostomium. Dorsal pore onset is perhaps just in advance. Setal tumescences are apparently not as extensive in current specimens (different preservation methods?) although setae ab were here partly occluded by tumidity. Zicsi (1972) did not note a typhlosole. DNA barcodes in Appendix 1 define species.

\section{Eisenia koreana (Zicsi, 1972)}

Eiseniella koreana Zicsi, 1972: 129.

Eisenia koreana: Easton, 1983; Blakemore, 2004: 98; 2008a: 39.

Type material. Budapest University, holotype Ei-8 and eleven paratypes registered as $\mathrm{Nr}$. 7000, from a brook bed behind Pyongyang Zoo (Mt. Taesong), North Korea.

Diagnosis. Size $45-52 \mathrm{~mm}$ by ca. $3 \mathrm{~mm}$ wide with $87-123$ segments. Grey green in life, pale laterally in 10-11. Pro-epilobic. Posterior squarish. Dorsal pores from 4/5. Setal distances stated as "ab=bc" in Zicsi (1972: 129) was likely a typing error for $a b=c d$, with $a a>b c$ and $d d<1 / 2 U$. Setae $a b$ tumid on $10,16,17,23,26-30$ plus on cd in 10 and 11 . Spermathecal pores in 9/10/11 dorsally but spermatophores also present. Clitellum 25-31, TP 27-29. Holandric with seminal vesicles in 9-12 (smaller in 10). Calciferous glands in 11 \& 12. Crop in 15-16, gizzard in 17-18.

Remarks. The stated reason Zicsi (1972: 131) attributed his species to the semi-aquatic genus Eiseniella Michaelsen, 1900 was for its inferred amphibian habitat, although it was later transferred (e.g., Easton, 1983) to Eisenia. And whereas Zicsi had primarily compared his taxon to "meroandrisch" Eisenia kucenkoi (Michaelsen, 1902) from "Ost-Turkestan" (=Xinjiang), a remarkable similarity to Eisenoides carolinensis from Appalachian southeastern USA was hitherto unrecognized. Both are squarish, with clitella and TPs having same range, the spermathecae are dorsal in 9/10/11 and calciferous glands are in $11 \& 12$. Similarity extends to a report (Gates 1956: 19) of spermatophores on an Ei. carolinensis worm attached at 25/26 and on 27, as also found by Zicsi (1972) in E. koreana at 22/23. Exact relationship of the taxa remains unclear.

Nearby at De-sang San, Zicsi (1972: 130) reported Eisenia nordenskioldi (Eisen, 1879) [corr. Blakemore (2008a: 39) ex nordenskiöldi non "nordenskioeldi" as per Blakemore (2004: 98)] also Aporrectodea caliginosa (Savigny, 1826).

\section{Eisenia sindo sp. nov. (Fig. 3)}

Material examined. Holotype, H IV0000246435 (sample \#3 mature, dissected; DNA WO25) (Fig. 3); paratype P1 IV00 00246436 (sample \#4 WO26) and paratypes IV0000246437 (12 matures, 5 juveniles, 5 immatures, and two hatchlings plus three fragments), all fixed in $75 \% \mathrm{EtOH}$. Collected 4 


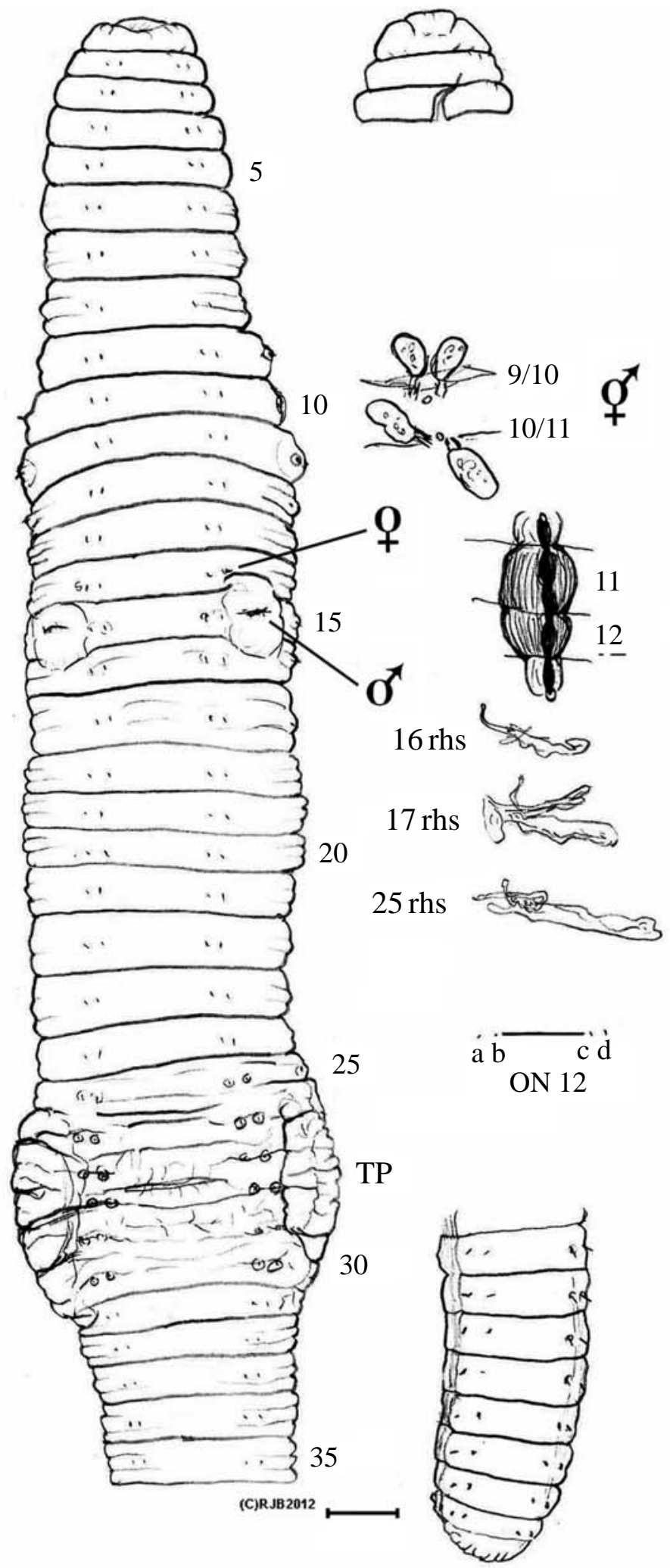

Fig. 3. Eisenia sindo: holotype (relaxed specimen IV0000246435) ventral view of anterior and prostomium; spermathecae in situ, sketch of calciferous glands in $11 \& 12$, vesiculate nephridia in 16, $17 \& 25$ rhs; posterior and actual setal ratios on 12 . rhs, right hand side; TP, tuberculae pubertates. Scale bar $=1 \mathrm{~mm}$. 
May 2012 by Blakemore RJ from mud beside creek between paddy fields just $200 \mathrm{~m}$ North of Sido bridge on Sindo Island, $37^{\circ} 53045^{\prime} \mathrm{N}, 126^{\circ} 43974^{\prime} \mathrm{E}$, Incheon.

Description. Body tapering, flattened in posterior (somewhat rectangular); palid colour with light brown pigment. Lengths 100-120 mm (H and P1). Segments 148-152 (P1 and H). Prostomium epilobic wide. Setae closely paired, obscured by tumidity ventrally on 16 and around clitellum; cd setae tumid on 10 and 11 laterally. Clitellum pale 25-31. TP lateral on ca. 26,27-29. Dorsal pores from 4/5. Spermathecal pores paired in $9 / 10 / 11$ near mid-dorsal line. Female pores on 14 , male pores on mounds in 15 .

Septa 5/6-8/9 slightly thick. Spermathecae inseminated, elongate in 9 and 10. Testis and funnels free, iridescent in 10 and 11. Seminal vesicles in 9-12 those in 10 small. Ovaries small in 13 , ovisacs vestigial in 14 . Hearts in 7-11. Nephridial bladders undeveloped or simple, mostly elongate sausage-shaped. Calciferous glands moniliform in $11 \& 12$. Crop in 16, muscular gizzard in 17-18. No typhlosole. Gut contains silty mud.

Remarks. Superficially similar to E. gaga, the current species is separated on small qualitative morphological differences (e.g., biometry, width of prostomium, shape of spermathecae, fewer nephridial bladders and lack of typhlosole) but mainly on their objective DNA barcode profiles (Appendix 1). Eisenia sindo differs from E. koreana in much the same way that E. gaga does whilst also complying superficially with Eisenoides carolinensis. However, due to proximity, it is more likely to be genetically closer to E. koreana than $E$. gaga is.

\section{DISCUSSION}

The North American genus Eisenoides Gates, 1969 [typespecies Allolobophora lönnbergi Michaelsen, 1894 corr. Blakemore (2008a: 42) to lonnbergi non "loennbergi"] was defined by Gates on its supposedly distinctive U-shaped nephridial bladders and, although these are somewhat variable, even intraspecifically (Blakemore, 2010), this is the only substantial morphological difference between similar genera since both Eisenia and Eiseniella are usually characterized by simple, sausage-shaped nephridial bladders.

Gates (1955: 10) said that seminal vesicles in segment 10 in one (or more?) of Eisenia pearsei Stephenson, 1933 types was all that distinguished it from Ei. carolinensis and that this did not warrant taxonomic recognition. Dr. Csaba Csuzdi (personal communication 21st-24th Feb 2012) informs that the single type of Ei. carolinensis in Hamburg, and the Ei. pearsei paratypes in London (its holotype being desiccated) have nephridial bladders that are indeed proclined, U-shaped (this information currently unpublished). Nephridial bladders were not explicitly described by Zicsi (1972), yet in the other two Eisenia species newly described herein they appear elongate or sausage-shaped, presumably in Eisenia koreana too, and solely on this feature they differ from Eisenoides carolinensis (Michaelsen, 1910). In other respects the present specimens and Eisenia koreana tolerably match earlier descriptions of Eisenoides carolinensis which is a remarkable morphological convergence co-incidence for taxa so widely separated geographically.

Support for their divergent separation is decided by DNA barcode comparison of types of E. gaga and of E. sindo with a voucher of Eisenoides carolinensis (Appendix 1). Dr. Csuzdi, a colleague of original author Dr. András Zicsi, also mentioned that primary types of Eisenia koreana from Mt. Taesong that he inspected in Budapest, due to their age and formol preservation, are unlikely to yield meaningful DNA data. Possibly E. sindo is closer with E. koreana, nevertheless, conspecificity can only be determined by DNA from its topotypes which are not currently accessible. In view of the DNA results for $E$. sindo and E. gaga, it is highly likely the three taxa form an Eisenia koreana species-complex with each speciating in and seeming to prefer localized moist or semiaquatic habitats.

\section{ACKNOWLEDGMENTS}

Dr. Csaba Csuzdi ex Hungarian Natural History Museum, Budapest is thanked for his contribution and comments. Some DNA sequencing was sub-contracted by TSP to Macrogen (WM material), others to Lee YH of Ehwa Womens' University, Seoul (WO material). All taxonomic work and decisions were by the senior author.

\section{REFERENCES}

Blakemore RJ, 2004. A provisional list of valid names of Lumbricoidea (Oligochaeta) after Easton, 1983. In: Advances in earthworm taxonomy (Annelida: Oligochaeta) (Eds., Moreno AG, Borges S). Universidad Complutense, Madrid, pp. 75120.

Blakemore RJ, 2008a. An updated list of valid, invalid and synonymous names of Criodriloidea and Lumbricoidea (Annelida: Oligochaeta: Criodrilidae, Sparganophilidae, Ailoscolecidae, Hormogastridae, Lumbricidae, Lutodrilidae). Chapter 10. A Series of Searchable Texts on Earthworm Biodiversity, Ecology and Systematics from Various Regions of the World. Version 2.0. Published under ICZN (1999: Art 8) then freely provided online [Internet]. COE Soil Ecology Research Group, Yokohama National University, Yokohama, Accessed 14 Feb 2012, <http://www.annelida.net/ 
earthworm/Lumbricidae.pdf $>$.

Blakemore RJ, 2008b. Preliminary checklist of Korean earthworms (original). Chapter 28. A Series of Searchable Texts on Earthworm Biodiversity, Ecology and Systematics from Various Regions of the World. Version 2.0 [Internet]. COE Soil Ecology Research Group, Yokohama National University, Yokohama, Accessed 14 Feb 2012, <http://www. annelida.net/earthworm/Korean.pdf>.

Blakemore RJ, 2010. Cosmopolitan earthworms: an eco-taxonomic guide to the peregrine species of the world. 4th ed. CDROM Monograph. VermEcology, Yokohama, pp. 1-750.

Blakemore RJ, Park TS, Seo HY, 2012. A new Korean earthworm (Oligochaeta: Megadrilacea: Megascolecidae). Zootaxa, 3368:256-262.

Easton EG, 1983. A guide to the valid names of Lumbricidae (Oligochaeta). In: Earthworm ecology: from Darwin to vermiculture (Ed., Satchell JE). Chapman \& Hall, London, pp. 475-487.

Gates GE, 1955. Notes on American earthworms of the family Lumbricidae. I-II. Breviora, 48:1-12.

Gates GE, 1956. Notes on American earthworms of the family Lumbricidae. III-VII. Bulletin of the Museum of Comparative Zoology at Harvard College, 115:1-46.
Gates GE, 1969. On two American genera of the earthworm family Lumbricidae. Journal of Natural History, 9:305-307.

Michaelsen W, 1894. Die Regenwurm-Fauna von Florida und Georgia, nach der Ausbeute des Herrn Dr. Einar Lönnberg. Zoologische Jahrbücher, Abteilung für Systematik, Jena, 8: 177-194.

Michaelsen W, 1900. Das Tierreich. Vol. 10. Vermes, Oligochaeta. Friedländer \& Sohn, Berlin, pp. 1-575.

Michaelsen W, 1902. Neue Oligochäten und neue Fundorte altbekannter. Mitteilungen aus dem Naturhistorischen Museum in Hamburg, 19:1-54.

Stephenson J, 1933. Oligochaeta from Australia, North Carolina, and other parts of the world. Proceedings of the Zoological Society of London, 1932:899-941.

Zicsi A, 1972. Zoological collecting by the Hungarian Natural History Museum in Korea. 7. Eiseniella koreana, eine neue Regenwurm-Art (Oligochaeta: Lumbricidae) aus Korea. Annales Historico-Naturales Musei Nationalis Hungarici, 64:129-132.

Received February 20, 2012 Revised April 17, 2012 Accepted April 24, 2012 
Appendix 1. DNA cytochrome c oxidase subunit 1 data and BLAST analysis.

$>$ WM1 Eisenia gaga holotype (INV 0000245509) (Fig. 1)

TAAGTGTTGATAGAGGATTGGGTCCCCTCCCCCCGCTGGATCAAAAAATGAAGTATTAAGATTTCGATCTGTTAGGAGTATGGTAATTGCCCCTGCT AAAACTGGTAAAGAAAGGAGGAGAAGAACTACTGTGATTACTACAGCTCAAACAAATAGGGGAATTCGTTCAAGTCGTAATCCTITTCATCGTATAT TAATAACAGTCGTAATGAAGTTGATGGCACCTAAGATTGAGGATGCACCTGCTAAGTGAAGAGAGAAAATGGCCAGATCTACTGAGGGGCCAGAG TGTGCGAGATTTCTAGATAGGGGGGGATAGACAGTTCATCCTGTTCCAGCTCCTTTTTCTACAGCAGCCGAGGATACTAATAAAATAAGAGATGGT GGAAGTAATCAAAATCTTATATTATTTAATCGGGGGAATGCCATGTCAGGGGCACCAAGTATTAGTGGGAGAAGCCAGTTTCCAAATCCTCCGATA AATACAGGTATCACAAGAAAAAAAATTATTACAAATGCATGCGCTGTAACGATAGTGTTATATAGTTGATCGCTACCTAAAAATGCTCCAGGTTGGC TTAACTCAATTCGGATTAGGAGGCTTATTCCTGCACCCACTATACCAGCTCAAACTCCTAGAATAAAATATA

$>$ WM2 E. gaga paratype1 (INV 0000245511 2nd dissected specimen)

AAGTGTTGATAGAGGATTGGGTCCCCTCCCCCCGCTGGATCAAAAAATGAAGTATTAAGATTTCGATCTGTTAGGAGTATGGTAATTGCCCCTGCT AAAACTGGTAAAGAAAGGAGGAGAAGAACTACTGTGATTACTACAGCTCAAACAAATAGGGGAATTCGTTCAAGTCGTAATCCTTTTCATCGTATAT TAATAACAGTAGTAATGAAGTTGATGGCACCTAAGATTGAGGATGCACCTGCTAAGTGAAGAGAGAAAATGGCCAGATCTACTGAGGGGCCAGAG TGTGCGAGATTTCTAGATAGGGGGGGATAGACAGTTCATCCTGTTCCAGCTCCTTTTTCTACAGCAGCTGAGGATACTAATAAAATAAGAGATGGT GGAAGTAATCAAAATCTTATATTATTTAATCGGGGGAATGCCATGTCAGGGGCACCAAGTATTAGTGGGAGAAGCCAGTTTCCAAATCCCCCGATA AATACAGGTATCACAAGAAAAAAAATTATTACAAATGCATGCGCTGTAACGATAGTGTTATATAGTTGATCGCTACCTAAAAATGCTCCAGGTTGGC TTAACTCAATTCGGATTAGGAGGCTTATTCCTGCACCCACTATACCAGCTCAAACTCCTAGAATAAAATA

$>$ WM3 E. gaga paratype (INV 0000245513 photographed specimen) (Fig. 2)

AATAAGTGTTGATAGAGGATTGGGTCCCCTCCCCCCGCTGGATCAAAAAATGAAGTATTAAGATTTCGATCTGTTAGGAGTATGGTAATTGCCCCT GCTAAAACTGGTAGAGAAAGGAGGAGAAGAACTACTGTGATTACTACAGCTCAAACAAATAGGGGAATTCGTTCAAGTCGTAATCCTTTTCATCGT ATATTAATAACAGTAGTAATGAAGTTGATGGCACCTAAGATTGAGGATGCACCTGCTAAGTGAAGAGAGAAAATGGCCAGATCTACTGAGGGGCCA GAGTGTGCGAGATTTCTAGATAGGGGGGGATAGACAGTTCATCCTGTTCCAGCTCCTTTTTCTACAGCAGCCGAGGATACTAATAAAATAAGAGAT GGTGGAAGTAATCAAAATCTTATATTATTTAATCGGGGGAATGCCATGTCAGGGGCACCAAGTATTAGTGGGAGAAGCCAGTTTCCAAATCCCCCG ATAAATACAGGTATCACAAGAAAAAAAATTATTACAAATGCATGCGCTGTAACGATAGTGTTATATAGTTGATCGCTACCTAAAAATGCTCCAGGTT GGCTTAACTCAATTCGGATTAGGAGGCTTATTCCTGCACCCACTATACCAGCTCAAACTCCTAGAATAAAATAT

$>$ WO25 Eisenia sindo holotype (IV0000246435) (Fig. 3)

ACCTTATACTTTATTCTTGGGGTTTGAGCCGGAATAGTAGGCGCTGGAATAAGCCTCTTAATCCGAATCGAGCTAAGACAGCCTGGAGCATTCCTG GGAAGAGACCAGCTATATAATACCATTGTTACAGCTCATGCGTTCGTAATAATCTTTTTCTTGTAATACCTGTATTTATTGGGGGGTTCGGCAACTG ACTTCTCCCATTAATATTGGGGGCTCCCGACATAGCATTCCCTCGTTTAAATAATATAAGATTCTGGCTACTTCCCCCTTCCCTTATTCTACTAGTCT CATCAGCAGCGGTTGAGAAAGGGGCGGGAACAGGTTGAACTGTGTACCCGCCCCTATCTAGAAATCTTGCACACGCTGGGCCATCAGTAGACCTG GCTATTTTCTCCCTTCATTTAGCGGGTGCGTCGTCTATTCTAGGAGCCATCAATTTTATCACTACAGTTATCAATATACGATGAAGAGGGTTACGTCT TGAACGAATTCCACTATTTGTGTGAGCTGTAGTAATTACTGTTGTTCTTCTTCTTCTCTCCCTACCAGTTCTAGCAGGAGCAATTACCATACTTCTAA CAGATCGAAACTTAAACACTTCATTCTTTGACCCCGCAGGAGGTGGAGATCCTATTCTTTATCAACATCTATT

$>$ WO26 E. sindo paratype P1 (IV0000246436)

CCTTATACTTTATTCTTGGGGTTTGAGCCGGAATAGTAGGCGCTGGAATAAGCCTCTTAATCCGAATCGAGCTAAGACAGCCTGGAGCATTCCTGG GAAGAGACCAGCTATATAATACCATTGTTACAGCTCATGCGTTCGTAATAATCTTTITTCTTGTAATACCTGTATTTATTGGGGGGTTCGGCAACTGA CTTCTCCCATTAATATTGGGGGCTCCCGACATAGCCTTCCCTCGTTTAAATAATATAAGATTCTGGCTACTTCCCCCTTCCCTTATTCTACTAGTCTC ATCAGCAGCGGTTGAGAAAGGAGCGGGAACAGGTTGAACTGTGTACCCGCCCCTATCTAGAAATCTTGCACACGCTGGGCCATCAGTAGACCTGG CTATTTTCTCCCTTCATTTAGCGGGTGCGTCATCTATTCTAGGAGCCATCAATTTTATCACTACAGTTATCAATATACGATGAAGAGGGTTACGTCTT GAACGAGTTCCACTATTTGTGTGAGCTGTAGTAATTACTGTTGTTCTTCTTCTTCTCTCCCTGCCAGTTCTAGCAGGAGCAATTACCATACTTCTAAC AGATCGAAACTTAAACACTTCATTCTTTGACCCCGCAGGAGGTGGGGATCCTATTCTTTATCAACATCTATTC

\section{Barcode results and BLAST conclusions}

1. BLASTn alignments: $>99 \%$ WM1 $=$ WM2 $=$ WM3, i.e., samples of same species.

2. megaBLAST of WM1 E. gaga:- no match better than $83 \%$ for various worm species on GenBank [GU013824 A. caliginosa and GU014206 "Acanthodrilidae sp." (sic!) by S. James], i.e., no similar species uploaded (therefore this presumably the first COI barcode report for Eisenia koreana or its ilk). GenBank upload imminent.

3. BLASTn alignment WM1 E. gaga vs. FJ214226 "Eisenia carolinensis" [sic] from Stateline, Louisiana partial COI data mined from GenBank:- no better than 79\% agreement, i.e., not same species-assuming correct identification of FJ214226.

4. megaBLAST of FJ214226 E. carolinensis:- best match $94 \%$ with an unidentified Romanian lumbricid (!), or $<85 \%$ similar to specimens identified as "Eisenia fetida," i.e., no named specimen closely similar to $E$. carolinensis yet uploaded on GenBank.

[Note: one website (http://bold.ala.org.au/index.php/display/item/GBAN2219-09/July, 2012) has this code and data identified as "Eisenia carolinensis" from Sao Paulo by Claudio Oliveira, but also recoded as "female" and "immature", so is highly dubious].

5. BLASTn alignment WM1 E. gaga vs. WO25 E. sindo:- Identities=530/646 (83\%), therefore the objective conclusion is these specimens are different species.

6. BLASTn alignment WO25 vs. WO26 E. sindo:- Identities=649/655 (99\%), i.e., tolerably same species.

7. megaBLAST WO25 E. sindo:- best match $83 \%$ of some Lumbricidae from GenBank, i.e., no similar species yet identified.

8. BLASTn alignment WO25 E. sindo vs. FJ214226 "Eisenia carolinensis":- Identities=516/652 (79\%), i.e., these are different species with above provisos. 